\title{
BS Indi: An enigmatic object in the Tucana association ${ }^{\star}$
}

\author{
E. W. Guenther ${ }^{1}$, E. Covino ${ }^{2}$, J. M Alcalá ${ }^{2}$, M. Esposito ${ }^{1,3}$, and R. Mundt ${ }^{4}$ \\ 1 Thüringer Landessternwarte Tautenburg, Sternwarte 5, 07778 Tautenburg, Germany \\ e-mail: guenther@tls-tautenburg.de \\ 2 INAF-Osservatorio Astronomico di Capodimonte, via Moiariello 16, 80131 Napoli, Italy \\ ${ }^{3}$ Università di Salerno, via S. Allende, 84081 Baronissi (SA), Italy \\ ${ }^{4}$ MPI für Astronomie, Königstuhl 17, 69117 Heidelberg, Germany
}

Received 1 November 2004 / Accepted 30 November 2004

\begin{abstract}
BS Ind (=HD 202947) is a young K0V star in the Tucana association. Photometric observations with the Hipparcos satellite show an eclipse-like light-curve with primary and secondary eclipse. The eclipsing binary has a period of 0.435338 days and a circular orbit. Our spectroscopic observations however show that the K0V primary is a single-line spectroscopic binary with a period of 3.3 years. The minimum mass of the invisible component is about $0.9 M_{\odot}$ which means that the mass of the companion is about the same as that of the primary. The first inspection of our FEROS spectra with a resolution of 48000 , as well as a CES spectrum with a resolution of 220000 shows no obvious companion. However, when the FEROS spectra are cross-correlated with an M-star, a secondary becomes visible as a broad peak in the cross-correlation function. The width and the position of this broad peak is variable on a short time. When phased to a period of 0.435338 days, the radial velocity variations of the broad peak show the characteristic sine-wave of a spectroscopic binary in a circular orbit. The best interpretation of this data is that the broad peak in the cross-correlation function is caused by an eclipsing binary consisting of two late- $\mathrm{K}$, or early-M stars with an orbital period of 0.435338 days. This is the eclipsing system. These two stars then orbit the K0V-primary with a period of 3.3 years. The assumption that BS Ind is a triple system consisting of a K0V star and two late-K, or early-M stars also explains the unusual brightness of the object and the near infrared excess. Thus, BS Ind is unique, as it contains by far the shortest-period young binary star, and these stars are eclipsing.
\end{abstract}

Key words. stars: individual: BS Ind - binaries: eclipsing - binaries: spectroscopic - stars: formation - stars: evolution

\section{Introduction}

In recent years a number of loose associations of young nearby stars have been identified. Among these new groups is the Tucana association (Torres et al. 2000) which has an age of about $30 \mathrm{Myr}$ and is located at a distance of about 45 to $60 \mathrm{pc}$. The Tucana association might be related to the close-by Horologium association (Zuckerman \& Webb 2000; Zuckerman et al. 2001) which has about the same distance and age. In total, about 50 stars have been identified in these associations. Because of the small distance, these associations are well suited for all kinds of studies of young stars. In the first step we survey these stars for the presence of visual companions (Neuhäuser et al. 2003). Similar to our survey for spectroscopic binaries of the TW Hydrae association (Torres et al. 2003), we have also surveyed the stars in the Horologium and Tucana associations.

* Based on observations obtained at the European Southern Observatory at La Silla, Chile in program 62.I-0418, 63.I-0096, 64.I-0294, 65.I-0012, 67.C-0155, 68.C-0292, 69.C-0207, 70.C-0163, 072.A-9012, 073.C-0355 and 67.C-0194.
BS Ind (HIP 105404, HD 202947) is one of the stars in the Tucana association. It is classified as a K0V star located at a distance of $46 \pm 3$ pc (Hipparcos, ESA 1997). With an absolute brightness of $M_{v}=5.59 \mathrm{mag}$ (Zuckerman et al. 2001), or $M_{v}=$ $5.54 \mathrm{mag}$ (Cutispoto et al. 2002), BS Ind is thus slightly above the main sequence. From our spectra, we derive an equivalent width of $0.176 \pm 0.020 \AA$ for the LiI 6707 line, which also confirms the young age of BS Ind. In summary, at first glance BS Ind seems to be a fairly normal low-mass star with an age of about 30 Myr located in the Tucana association.

\section{The light-curve of BS Ind}

The Hipparcos catalogue lists BS Ind as an eclipsing binary with a period of 0.435338 days (Hipparcos, ESA 1997). Is it really an eclipsing binary? The phase-folded light-curve of BS Ind in fact looks like that of a typical eclipsing binary, with primary and secondary eclipses (Fig. 1). Additionally, the secondary eclipse appears, though barely above the noise, at phase 0.5 , as is expected for a companion on a circular orbit. The depth of the primary eclipse is $0.26 \pm 0.01 \mathrm{mag}$, and that 


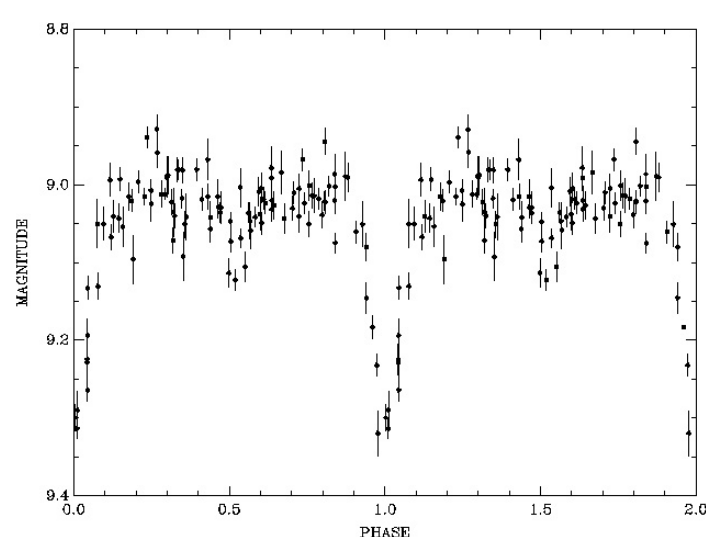

Fig. 1. Hipparcos light-curve phase-folded with the period from the light-curve of 0.435338 days. The primary eclipse is clearly visible, and the secondary eclipse is barely visible.

of the secondary $0.10 \pm 0.01 \mathrm{mag}$ in the Hipparcos $V$-band. Although the data is quite noisy, we estimate the time difference between the first and the fourth contact as $1.5 \pm 0.2 \mathrm{~h}$, and the time difference between the second and the third contact as $0.5 \pm 0.2 \mathrm{~h}$. A spectrum taken by Cutispoto et al. (2002), shows a double-line emission core in a Ca II K-line but not in the LiI 6707-line. The implication of this observation will be discussed in Sect. 4.

In principle stellar spots can mimic an eclipsing binary light-curve (Joergens et al. 2001). The question thus arises of whether we can exclude this possibility. A spot should be visible half of the time, if the star is viewed close to equator-on, and even longer if it is viewed more pole-on. This is certainly not what we observe. Secondly, from our spectra we derive a $v \sin i$ of $13.0 \pm 0.4 \mathrm{~km} \mathrm{~s}^{-1}$ which is in excellent agreement with the value from the literature (Zuckerman \& Webb 2000; Cutispoto et al. 2002). If the light-curve were caused by spots, the true rotation period would have to be 0.435338 days, which implies that the inclination should be less than 10 degrees. If the inclination would be that low, it is hard to imagine how spots could produce an 0.3 mag dip in the light-curve. The spot would have to cover more than $20 \%$ of the stellar surface and it would need to be hidden most of the time, thus it would have to be located close to the equator. However, a spot located close to the equator on a star that is viewed almost pole-on can not produce a rapid decline like the one observed (Fig. 1).

There is further evidence against the hypothesis of such a short rotation period: like other stars of the same age, BS Ind is an X-ray source. The logarithmic X-ray luminosity varies between 29.4 and $30.4 \log \left(\mathrm{erg} \mathrm{s}^{-1}\right)$. This value can be compared to the $\log$ of the mean X-ray luminosity of $28.94 \pm$ $0.06 \log \left(\mathrm{erg} \mathrm{s}^{-1}\right)$ for K-stars in the Pleiades which are older than BS Ind, and $29.78 \pm 0.10 \log \mathrm{erg} \mathrm{s}^{-1}$ for WTTS in the Taurus star-forming region which are younger than BS Ind (Stelzer \& Neuhäuser 2000). The X-ray brightness of BS Ind thus is what is expected for a $\mathrm{K}$-star of this age. Furthermore, the $\log$ of X-ray luminosity is $29.7 \log \left(\mathrm{erg} \mathrm{s}^{-1}\right)$ for stars with a rotation period of 4 days in the Taurus star-forming region and $29.2 \log \left(\mathrm{erg} \mathrm{s}^{-1}\right)$ for stars with a rotation period of 4 days in the Pleiades, respectively. Thus, the X-ray brightness of BS Ind is typical for a $\mathrm{K}$-star of the same age that has a rotation period of about 4 days. A rapidly rotating star should be much brighter at X-rays. The hardness ratios are also within the range of other stars in Tucana (Kastner et al. 2003). Thus, the X-ray brightness and the $v \sin i$ both support the idea that BS Ind is an eclipsing system and not a very rapidly rotating star with huge, unevenly distributed, photospheric spots.

\section{The long-period orbit}

In order to solve the orbit of BS Ind, we observed the star with the ESO echelle spectrograph FEROS (Fiber-fed Extended Range Optical Spectrograph) on the $1.5-\mathrm{m}$, and 2.2-m-telescope at La Silla. The spectra cover the wavelength region between about $3600 \AA$ and $9200 \AA$, and have a resolution of $\lambda / \Delta \lambda=48000$. The standard MIDAS pipeline for FEROS was used for bias subtraction, flat-fielding, scattered light removal, echelle order definition and extraction, and wavelength calibration of the spectra. Precise measurements of the radial-velocity (RV) have then been carried out by cross-correlating the object spectra with spectra of the RV standard HR 5777 observed with the same instrument using the IRAF RV package. The RV of HR 5777 is known to be stable, and has been determined with an accuracy of $55 \mathrm{~ms}^{-1}$ (Murdoch 1993). We have observed HR 5777 in every observing run and find that the scatter of our measurements is $80 \mathrm{~ms}^{-1}$, which is consistent with the expected errors of the measurements. The cross-correlation function between BS Ind and HR 5777 shows only one peak. Because of the smaller signal to noise ratio and the larger $v \sin i$, the errors on the RV-measurements of BS Ind are often larger (see Table 1). We also took one spectrum of BS Ind with the CES - Coude Echelle Spectrometer at the 3.6-m-telescope at La Silla in the wavelength region between 6402-6445 $\AA$ with the Highresolution image slicer. This instrument has a resolution, $\lambda / \Delta \lambda$, of 220000 . The spectrum of BS Ind was exposed for twice $1200 \mathrm{~s}$. Standard IRAF routines were used for the data analysis.

Table 1 gives the RV measurements obtained for BS Ind. An eclipsing M0V-star would have a mass of about $0.5 M_{\odot}$ which will induce RV-variations with an amplitude of more than $200 \mathrm{~km} \mathrm{~s}^{-1}$, if it is orbiting the primary star with a period of 0.435338 days, and using $M_{1}=0.8 M_{\odot}$ for the primary. The much smaller amplitude observed already indicates that either we observed the object incidentally always at the same phase, or the mass of the companion is much lower, or the orbital period is much longer. Figure 2 shows the RV measurements phase-folded with the period 0.435338 days. The dashed line is for $f(m)=\frac{M_{2}^{3} \sin ^{3} i}{\left(M_{1}+M_{2}\right)^{2}}=0.07 M_{\odot}$, which corresponds to $K_{1}=116 \mathrm{~km} \mathrm{~s}^{-1}$. As a systemic velocity we use the average RV of stars in the Tucana association of $20.6 \pm 4.6$, and we set $\sin i=1$, because the system is supposed to be eclipsing. Clearly, this curve does not fit the data. Note that errorbars of our RV-measurements are so small that they cannot be seen in the plot. Even if we assume a much lower mass, the $\mathrm{RV}$-measurements still cannot be reproduced with this period. BS Ind clearly is not a simple eclipsing binary. 
Table 1. Radial velocity measurements and corresponding rms errors versus Heliocentric Julian Day. The first column indicates the running number of the spectrum. (* taken from Cutispoto et al. 2002).

\begin{tabular}{|c|c|c|}
\hline No. & HJD & $\mathrm{RV}\left[\mathrm{km} \mathrm{s}^{-1}\right]$ \\
\hline Cutispoto $*$ & 2449253.5 & $31.0 \pm 1.1$ \\
\hline Cutispoto $*$ & 2449597.5 & $9.0 \pm 1.8$ \\
\hline FEROS No. 1 & 2451737.9193 & $32.53 \pm 0.04$ \\
\hline FEROS No. 2 & 2452031.9386 & $8.54 \pm 0.10$ \\
\hline FEROS No. 3 & 2452089.8273 & $5.88 \pm 0.20$ \\
\hline FEROS No. 4 & 2452093.8171 & $6.74 \pm 0.05$ \\
\hline FEROS No. 5 & 2452093.8513 & $6.72 \pm 0.05$ \\
\hline FEROS No. 6 & 2452093.8823 & $6.75 \pm 0.06$ \\
\hline FEROS No. 7 & 2452093.9113 & $7.14 \pm 0.09$ \\
\hline FEROS No. 8 & 2452093.9404 & $6.95 \pm 0.11$ \\
\hline FEROS No. 9 & 2452093.9532 & $6.79 \pm 0.08$ \\
\hline CES & 2452105.9038 & $6.91 \pm 0.16$ \\
\hline FEROS No. 10 & 2452372.9120 & $5.76 \pm 0.10$ \\
\hline FEROS No. 11 & 2452372.9224 & $5.62 \pm 0.05$ \\
\hline FEROS No. 12 & 2452373.8833 & $5.50 \pm 0.05$ \\
\hline FEROS No. 13 & 2452373.8961 & $5.22 \pm 0.08$ \\
\hline FEROS No. 14 & 2452373.9073 & $4.86 \pm 0.09$ \\
\hline FEROS No. 15 & 2452384.9085 & $5.66 \pm 0.07$ \\
\hline FEROS No. 16 & 2452385.9122 & $5.71 \pm 0.13$ \\
\hline FEROS No. 17 & 2452710.8900 & $7.87 \pm 0.14$ \\
\hline FEROS No. 18 & 2452717.9110 & $8.42 \pm 0.05$ \\
\hline FEROS No. 19 & 2452723.9049 & $8.65 \pm 0.06$ \\
\hline FEROS No. 20 & 2453096.9020 & $13.55 \pm 0.11$ \\
\hline FEROS No. 21 & 2453104.87233 & $12.74 \pm 0.09$ \\
\hline FEROS No. 22 & 2453105.89532 & $12.97 \pm 0.05$ \\
\hline FEROS No. 23 & 2453137.88899 & $11.03 \pm 0.05$ \\
\hline FEROS No. 24 & 2453137.89652 & $10.99 \pm 0.05$ \\
\hline FEROS No. 25 & 2453137.90404 & $10.24 \pm 0.14$ \\
\hline FEROS No. 26 & 2453137.90475 & $10.32 \pm 0.05$ \\
\hline FEROS No. 27 & 2453162.87508 & $9.56 \pm 0.10$ \\
\hline FEROS No. 28 & 2453162.88265 & $9.59 \pm 0.06$ \\
\hline FEROS No. 29 & 2453168.78650 & $10.34 \pm 0.06$ \\
\hline FEROS No. 30 & 2453168.79417 & $10.22 \pm 0.06$ \\
\hline FEROS No. 31 & 2453168.83513 & $9.76 \pm 0.07$ \\
\hline FEROS No. 32 & 2453168.84271 & $9.69 \pm 0.06$ \\
\hline
\end{tabular}

As can clearly be seen from Table 1, BS Ind does show significant RV-variations. However, on HJD 2452093 we took 6 spectra within $3.3 \mathrm{~h}$. The scatter of $0.15 \mathrm{~km} \mathrm{~s}^{-1}$ of these datapoints is fully consistent with the accuracy of the measurements, and already excludes a very short period, massive companion. Spectra taken from HJD 2452710 to HJD 2452723 differ also only by $0.78 \pm 0.15 \mathrm{~km} \mathrm{~s}^{-1}$, and spectra taken between HJD 2453096 and HJD 2453105 only by $0.85 \pm$ $0.15 \mathrm{~km} \mathrm{~s}^{-1}$. This indicates that a period of the radial velocityvariations below 10 days is not possible. If we add in the two data-points from Cutispoto et al. (2002), we find

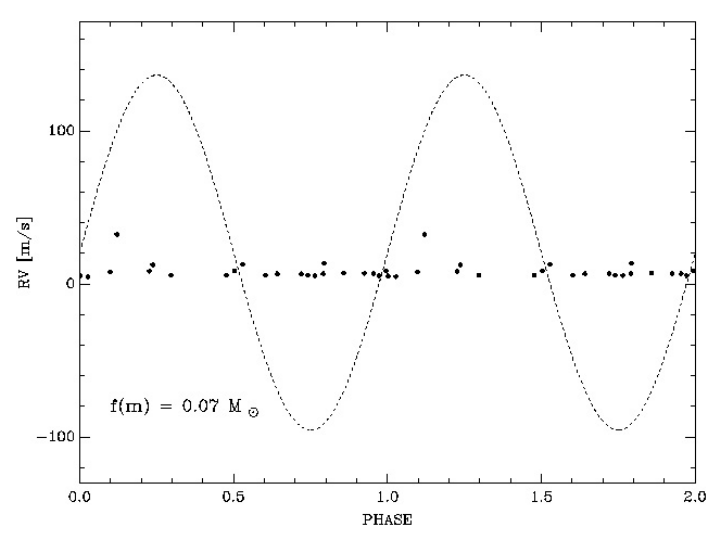

Fig. 2. Radial velocity measurements phase-folded with the period from the light-curve of 0.435338 days. The dashed line is for $f(m)=$ $\frac{M_{2}^{3} \sin ^{3} i}{\left(M_{1}+M_{2}\right)^{2}}=0.07 M_{\odot}$. For the systems velocity the average RV of stars in the Tucana association of $20.6 \pm 4.6$ is used $\mathrm{km} \mathrm{s}^{-1}$. Clearly, this curve does not fit the RV-measurements. BS Ind cannot be a simple eclipsing binary.

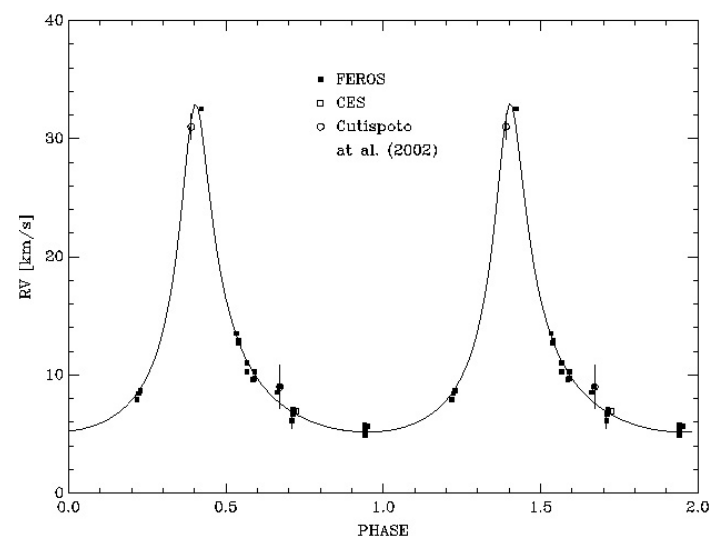

Fig. 3. The most likely orbital period is 3.3 years.

Table 2. Orbit of BS Ind.

\begin{tabular}{ll}
\hline \hline Period & $1223 \pm 30$ days \\
Eccentricity & $0.60 \pm 0.05$ \\
$\omega$ & $-6 \pm 2^{\circ}$ \\
$R_{0}$ & $10.8 \pm 0.2 \mathrm{~km} \mathrm{~s}^{-1}$ \\
$f(m)=\frac{M_{2}^{3} \sin ^{3} i}{\left(m_{1}+m_{2}\right)^{2}}$ & $0.273 \pm 0.06 M_{\odot}$ \\
$a \sin i$ & $2.7 \pm 0.2 \mathrm{AU}$ \\
$m_{1}$ & $0.8 \pm 0.1 M_{\odot}$ \\
$m_{2}$ & $0.9 \pm 0.1 M_{\odot}$ \\
periastron passage [JD] & $2450489 \pm 30$ \\
\hline
\end{tabular}

a period of 3.3 years, and a mass function $f(m)=\frac{M_{2}^{3} \sin ^{3} i}{\left(m_{1}+m_{2}\right)^{2}}$ of $0.273 \pm 0.06 M_{\odot}$ which implies a minimum mass of the companion of $0.9 \pm 0.1 M_{\odot}$ assuming a mass of the primary of $0.8 \pm 0.1 M_{\odot}$. The resulting fit is shown in Fig. 3 and the orbital parameters derived are given in Table 2 . The systemic velocity, $R_{0}=10.8 \pm 0.2 \mathrm{~km} \mathrm{~s}^{-1}$ is notably different from the average RV of stars in the Tucana association of $20.6 \pm 4.6 \mathrm{~km} \mathrm{~s}^{-1}$. 


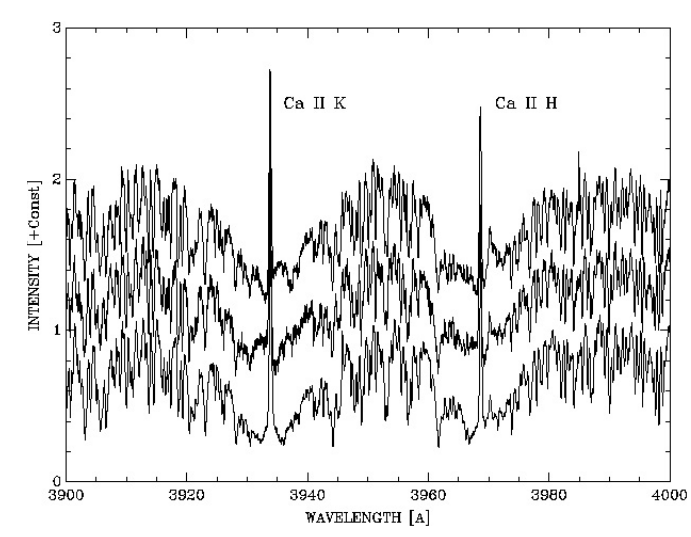

Fig. 4. A spectrum taken by Cutispoto et al. (2002) shows a doublepeaked emission line structure in the Ca II K-line. In here we show as examples from top to bottom spectrum No. 4, No. 15, and No. 20. One emission core is seen but nothing obvious is seen from the companions.

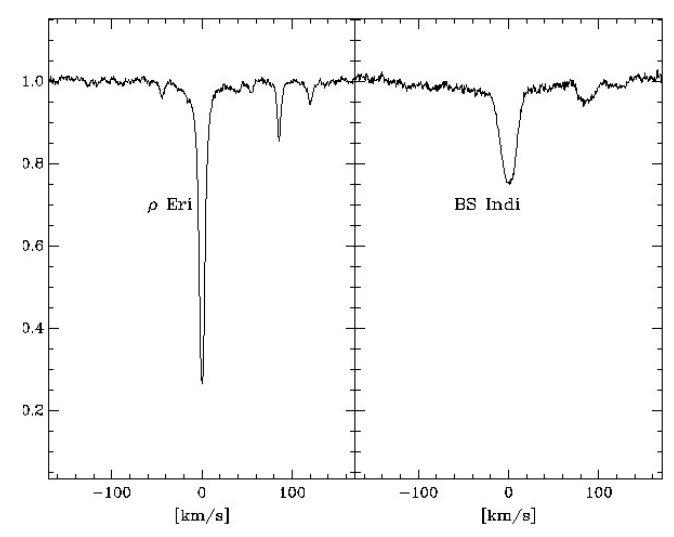

Fig. 5. CES spectrum of the FeI 6430.856 line of the K5V star $\rho$ Eri, and BS Ind. The spectral line of BS Ind is broader, because of the larger $v \sin i$ but there is no obvious trace of the companions in this spectrum.

\section{The detection of the two eclipsing stars}

The best explanation for the fact that the K0V-primary is a long-period, single-line, spectroscopic binary, and the light-curve shows a short period eclipsing system is that BS Ind is a triple system. The eclipsing components are two late-K, or early-M stars orbiting each other with a period of 0.435338 days, and this binary system orbits the K0V-primary with a period of 3.3 years. In order to prove this scenario the two eclipsing stars have to be detected in the spectrum.

As we pointed out before, when we cross-correlate the FEROS spectra with the K1IV-star HR 5777 there is only one peak. Figure 4 shows three of our FEROS spectra of this region. In none of these spectra we see any obvious companion. On the other hand, the spectral region around the Ca II K-line is very crowded and thus might not be the best region to look for a faint companion. As mentioned above, a spectrum taken by Cutispoto et al. (2002) show a double-line structure in the Ca II K-line. Because this feature was observed only once, it is apparently highly variable. Since the Ca II -lines are prominent during flare-events it is possible that the second peak was caused by a flare-event. This idea is also consistent with the

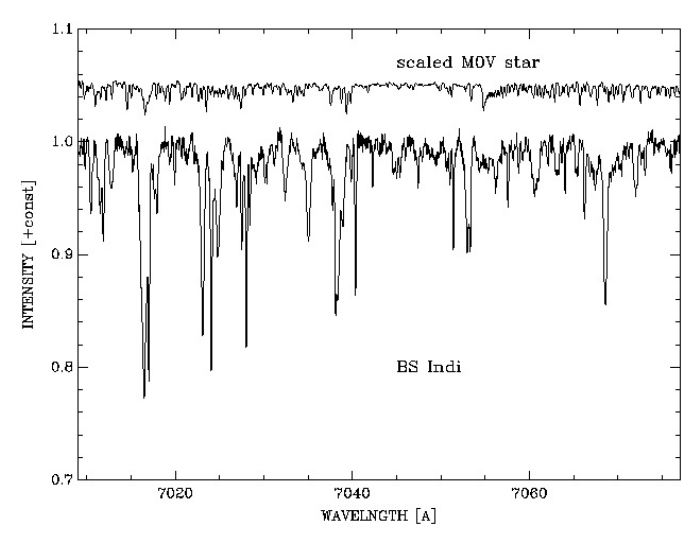

Fig. 6. The lower spectrum is the averaged of BS Ind. Averaged were all spectra from No. 10 to No. 19. since these have roughly the same RV. Above is a spectrum of an M0V star that is 2.9 mag fainter than BS Ind (shifted by 0.05). The TiO band-head at $7055 \AA$ is the strongest feature in the spectrum of an M-dwarf. Even the averaged spectrum of BS Ind does not allow to exclude, or confirm the presence of such a companion.

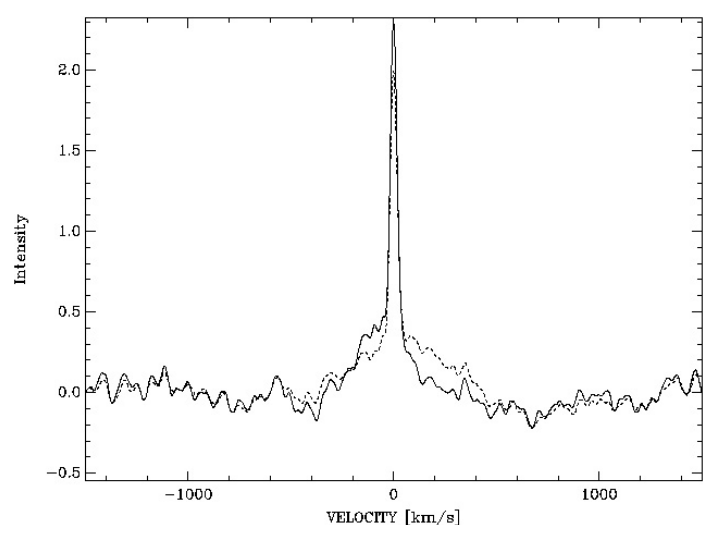

Fig. 7. Two cross-correlation functions. In both cases BS Ind is crosscorrelated with an M0III-star. Apart from the sharp peak which is due to the primary KOV star, a broad feature is seen. This is the signature of the two eclipsing stars. Unfortunately the $v \sin i$ of the tow eclsiping stars is so large that the two are not separated. Neverthless, we find that the broad peak changes its positions.

absence of a double-line peak in the Li I 6707 line. The wavelength region between 6402-6445 $\AA$ contains a number of unblended and well separated spectral lines and is thus wellsuited for the search of the secondary component. Figure 5 shows a CES spectrum with a resolution of $\lambda / \Delta \lambda=220000$ of the FeI 6430.856 line of the K5V star $\rho$ Eri together with spectrum of BS Ind. Again there is no obvious sign of the companions. The strongest feature in the spectrum of an early M-star are the TiO-band-heads. Figure 6 shows the spectrum of BS Ind together with a properly scaled M star. Even the averaged spectrum of BS Ind does not allow to exclude, or confirm the presence of such a companion.

As a last step we finally cross-correlated the FEROS spectra of BS Ind with the M0III star HR 6056. In this case we did detect a broad peak (Fig. 7). This broad peak is observed in all our FEROS spectra when cross-correlated with the M-star. The position and the width of this peak is variable, and we regard it as the signature of the two eclipsing early-M, or late-K K stars. 


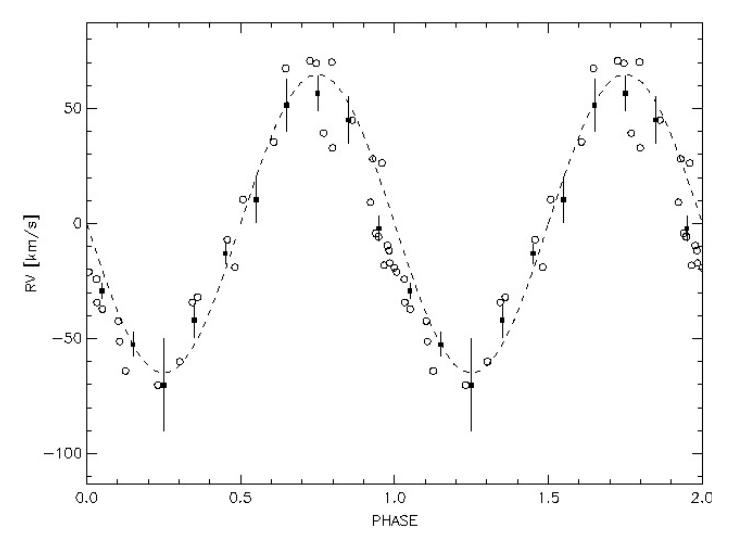

Fig. 8. The RV curve derived for the broad peak, after subtracting the 3.3 year orbit, and after phase-folding the data to the period of 0.435338 days. The filled dots are the phase averaged data points, and the open circles are the individual measurements. The phase is the same as in Fig. 1. The eclipse in fact appears, at RV $=0$, and before the primary eclipse the spectrum is red-shifted, just as it should be. Thus, there is clear evidence that the position of the broad peak varies with a period of 0.435338 days. The semi-amplitude is about $65 \mathrm{~km} \mathrm{~s}^{-1}$.

After subtracting the 3.3 years orbit from this data, and phasefolding it with the 0.435338 day period, we find in fact a sinusoidal variation of the RV (Fig. 8). The phase in this figure is the same as in Fig. 1. The eclipse thus in fact appears, at RV $=0$, and before the primary eclipse the spectrum is red-shifted, just as it should be for an eclipsing binary. The two eclipsing stars appear only as one broad peak, because the $v \sin i$ of stars are of the order of $100 \mathrm{~km} \mathrm{~s}^{-1}$. This is because the orbital period and the rotational period of the two stars is the same, because the orbital period is so short. Because of the large $v \sin i$ the lines of the two stars are not separated, and because one is brighter than the other, the width and the position of the broad peaks in the cross-correlation function vary. This is fully consistent with the light-curve, which also indicates that one of the eclipsing stars is brighter. Unfortunately this implies that amplitude of the radial-velocity variations of the broad peak is only the change of the barycenter of the blended cross-correlation functions of the two stars. The true amplitude of the RV-variations is larger. This implies that although the $\mathrm{B}$ and $\mathrm{C}$ component of BS Ind are eclipsing, we cannot determine their true masses. We thus conclude that BS Ind in fact consist of two eclipsing stars orbiting each other with a period of 0.435338 days, and this system orbits the K0V-primary with a period of 3.3 years.

\section{Conclusions}

The photometry from the Hipparcos satellite indicates the presence of two eclipsing stars orbiting each other with a period of 0.435338 days. BS Ind thus has a lot in common with the multiple star HD 97770, which also contains two eclipsing late type stars (K4/5 V star and a K5V star), with an almost similar orbital period of $0.476533 \pm 0.000033$ days (Cutispoto et al. 1997). Like BS Ind, HD 97770 shows flares and is bright in $\mathrm{X}$-rays. The main difference is that BS Ind is possibly younger than HD 97770. The light-curve of BS Ind cannot be explained by a spot on the star. The RV measurements clearly indicate that BS Ind is not a short-period eclipsing object but an SB1-binary with an orbital period of 3.3 years. The mass of the secondary component is, however, about one solar mass. The cross-correlation function does show, apart from the sharp peak due to the K0V star, a very broad peak which varies significantly in RV and width. This can best be explained as the signature of two stars orbiting each other. The two eclipsing stars, whose total mass is about $0.9 M_{\odot}$, are thus either late-K or early-M stars. Both the light-curve and the cross-correlation function indicate that the brightness of the two eclipsing stars is slightly different. The presence of the two eclipsing stars also explains the total brightness of the BS Ind-system in the optical and in the near infrared regime. Assuming that the two eclipsing stars have about $0.5 M_{\odot}$, they would be roughly M0V stars and thus together would be 2.1 mag fainter than the primary in the visible. If this were true, we would expect a decrease in brightness by $0.15 \mathrm{mag}$ if one of the stars is eclipsed. This value is is in the middle between the primary and secondary eclipse. In reality one of the eclipsing stars thus must be a slightly brighter and the other a slightly fainter. The system can best be disentangled once we know the true brightness of the primary and the eclipsing system separately, which will be possible once VLTI measurements are obtained. If we subtract the flux of two M0V-stars from the measured absolute brightness of the whole BS Ind-system, we find that the K0V-primary has an absolute brightness of $M_{v}=5.7 \pm 0.1$ and would thus be essentially on the main-sequence, like the other stars in Tucana. If the system would consist of two M0V stars and a K0V star it should be 7.2, 6.6, and $6.5 \mathrm{mag}$ in the $J, H$, and $K$-band, respectively instead of 7.5, 7.0 and 7.0 for single K0V-star. This is again in perfect agreement with the 2MASS point source catalogue which gives values of $7.184 \pm 0.026$, $6.699 \pm 0.031$, and $6.574 \pm 0.024 \mathrm{mag}$ for $J, H$, and $K$-band for BS Ind, respectively.

Thus, the fact that BS Ind is a triple system consisting of a KOV star and two stars with a spectral type of about M0V not only explains our spectroscopic observations and the Hipparcos light-curve, but also the optical and near infrared fluxes. Thus, BS Ind is by far the shortest period young lowmass binary system known. Unfortunately, the radial-velocities of the two eclipsing stars cannot be measured individually, and thus the masses of these stars cannot be measured individually. However, the fact that there is a young binary with an orbital period of only 0.435338 days raises the question of how such a system could have formed. The possible observation of a flare by Cutispoto et al. (2002) also fits nicely into the picture that the $\mathrm{K} 0$-primary is orbited by two late-type stars of very short period. Such binaries are active and thus show flare-activity.

Addtionally, the separation between the primary and the two eclipsing stars is large enough to be resolved with VLTI. In this way it will be possible to obtain the true mass of the primary and the total mass of the two eclipsing stars.

Acknowledgements. We are grateful to the support group of the 1.5-m, and 2.2-m-telescopes at La Silla, especially Lisa Germany, Olivier Hainaut, Emanuela Pompei, John Pritchard, Linda Schmidtobreick, Fernando Selman, Rolando Vega, Erich Wenderoth, 
for helping us with the observations. We would also like to thank the referee G. Cutispoto for sending us his spectra of BS Ind and for helping us improve the manuscript. This research has made use of the SIMBAD database, operated at CDS, Strasbourg, France.

\section{References}

Cutispoto, G., Kuerster, M., Messina, S., Rodonó, M., \& Tagliaferri, G. 1997, A\&A, 320, 586

Cutispoto, G., Pastori, L., Pasquini, L., et al. 2002, A\&A, 384, 491

ESA 1997, The Hipparcos and Tycho Catalogues (ESA SP-1200) (Noordwijk: ESA)

Guenther, E. W., Lehmann, H., Emerson, J. P., \& Staude, J. 1999, A\&A, 341, 768
Joergens, V., Guenther, E., Neuhäuser, R., Fernández, M., \& Vijapurkar, J. 2001, A\&A, 373, 966

Kastner, J. H., Crigger, L., Rich, M., \& Weintraub, D. A. 2003, ApJ, 585,878

Torres, G., Guenther, E. W., Marschall, L. A., et al. 2003, AJ, 125, 825

Murdoch, K. A., Hearnshaw, J. B., \& Clark, M. 1993, 413, 349

Neuhäuser, R., Guenther, E. W., Alves, J., et al. 2003, AN, 324, 535

Stelzer, B., \& Neuhäuser, R. 2000, A\&A, 361, 581

Torres, C. A. O., da Silva, L., Quast, G. R., de la Reza, R., \& Jilinski, E. $2000, \mathrm{AJ}, 120,1410$

Zuckerman, B., \& Webb, R. A. 2000, ApJ, 535, 959

Zuckerman,B., Song, I., \& Webb, R. A. 2001 ApJ, 559, 388 